\title{
Optical trapping using all-dielectric silicon nanoantennas with ultra-low heat generation
}

\author{
Zhe Xu' ${ }^{1}$, Wuzhou Song ${ }^{1,2}$, and Kenneth B. Crozier ${ }^{1,3^{*}}$ \\ ${ }^{1}$ School of Physics, University of Melbourne, Victoria 3010, Australia \\ ${ }^{2}$ School of Materials Science and Engineering, Huazhong University of Science and Technology, Wuhan 430074, P. R. China \\ ${ }^{3}$ Department of Electrical and Electronic Engineering, University of Melbourne, Victoria 3010, Australia \\ Corresponding Author e-mail address: kenneth.crozier@unimelb.edu.au
}

\begin{abstract}
Silicon nanoantennas are used to trap polystyrene nanospheres (20 nm diameter). Fluorescence microscopy is used to monitor trapped particle position as a function of time. The nanoantennas produce subwavelength field enhancement with negligible heat generation.

OCIS codes: (140.7010) Laser trapping; (310.6628) Subwavelength structures, nanostructures;
\end{abstract}

\section{Introduction}

Plasmonic nanoantennas permit the trapping of nanoscale objects [1], but the problem of Joule heating must be considered [2]. In Ref [3], for example, heating was reduced $~ 100$-fold by integrating a heat sink structure with a plasmonic nanotweezer. The need for reduced heating has been recognized for many applications of nanoantennas (and not just nanotweezers), prompting theoretical studies into all-dielectric nanoantennas [4]. Recently, silicon (Si) dimer antennas on glass substrates were reported for surface enhanced fluorescence and Raman scattering [5]. Here, we demonstrate the trapping of nanospheres (NSs) with diameters of 20 and $100 \mathrm{~nm}$ in water using a novel alldielectric nanoantenna comprising a pair of Si cylinders surrounded by an Si ring, all on an Si substrate (Fig.1a). The nanoantennas are fabricated by electron beam lithography and reactive ion etching. Our device offers important advantages compared to previous nanoantennas for optical trapping, which have been based on metals. First, at the wavelength employed here $(\lambda=1064 \mathrm{~nm})$, the real part of the permittivity of $\mathrm{Si}$ is much larger than its imaginary part, greatly reducing heating. Second, the high thermal conductivity of Si means that any heat generated is conducted quickly into the Si substrate. Here, we present simulations showing sub-wavelength field confinement with insignificant heat generation. We present trapping experiments with NSs with diameters of 20 and $100 \mathrm{~nm}$.
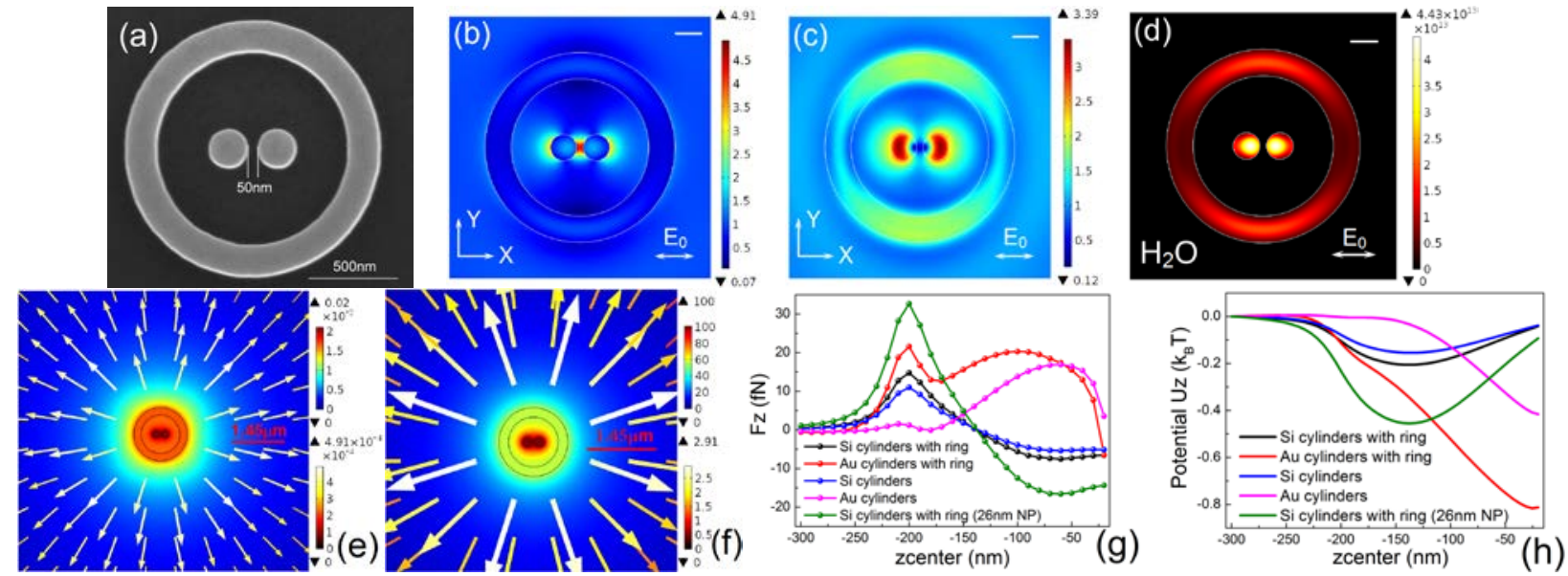

Fig.1. (a) SEM top-view image of Si nanoantenna. Each nanoantenna consists of two identical Si cylinders with diameters of $200 \mathrm{~nm}$, heights of $200 \mathrm{~nm}$ and a $50 \mathrm{~nm}$ gap in between, surrounded by an Si ring, all on an Si wafer substrate. Ring has inner and outer radii of 0.525 and $0.725 \mu \mathrm{m}$, respectively, and height $200 \mathrm{~nm}$. (b) Electric field ( $\left.\left|\mathrm{E} / \mathrm{E}_{0}\right|\right)$, (c) magnetic field $\left(\left|\mathrm{H} / \mathrm{H}_{0}\right|\right)$ enhancement, and (d) heat power dissipation density $\left(W / \mathrm{m}^{3}\right)$ in center plane $(z=-100 \mathrm{~nm})$ of nanoantenna. Scale bar: $200 \mathrm{~nm}$. Steady-state temperature increase ( $\Delta T$ in $K$, upper colorbar) in (e) Si antenna and (f) Au antenna. Colored arrows show water convection velocity field $(\mathrm{nm} / \mathrm{s})$. Magnitude of velocity is represented by length and color of arrow (lower colorbars). (g) Force and (h) trapping energy exerted on particle $(n=1.6)$. For green curve, particle has diameter $26 \mathrm{~nm}$. For other curves, diameter is $20 \mathrm{~nm}$. For panels d-f, illumination intensity is $\mathrm{I}_{0}=5 \mathrm{~mW} / \mu \mathrm{m}^{2}$. For panels $\mathrm{g} \& \mathrm{~h}, \mathrm{I}_{0}=9.5 \mathrm{~mW} / \mu \mathrm{m}^{2}$.

\section{Electromagnetic simulations}

We simulate our Si nanoantennas (Fig. 1a) using COMSOL Multiphysics. The Si nanoantennas are covered with water. Plane wave illumination ( $x$-polarized, $\lambda=1064 \mathrm{~nm}$ ) is incident from the water side. In Fig. 1b and 1c, we show the electric and magnetic field enhancements respectively on a plane that is halfway through the nanoantenna (i.e. $100 \mathrm{~nm}$ from the Si substrate surface). In Fig.1d, we plot the power dissipation density, again in a plane halfway 
through the nanoantenna, at an illumination intensity that might be typical for experiments $\left(5 \mathrm{~mW} / \mu \mathrm{m}^{2}\right)$. The steadystate local temperature and water convection velocity field around Si nanoantennas are also studied. As shown in Fig.1e, the simulations predict the maximum temperature rise and the maximum thermally-induced transverse fluid convection velocity are $\sim 0.02 \mathrm{~K}$ and $\sim 4.91 \times 10^{-4} \mathrm{~nm} / \mathrm{s}$, respectively, on the plotted cross-section. These are both very small. Fig.1f shows the calculation repeated for a gold ( $\mathrm{Au}$ ) nanoantenna of the same dimensions under the same illumination conditions. The Au nanoantenna is on a glass substrate. It can be seen that the maximum temperature rise $(\sim 100 \mathrm{~K})$ and transverse fluid velocity $(\sim 2.91 \mathrm{~nm} / \mathrm{s})$ are drastically higher than those predicted for the $\mathrm{Si}$ nanoantenna. We next find the optical forces (Fig.1g, in vertical direction) and potential energy (Fig.1h) exerted on a polystyrene nanosphere (diameter of 20 or $26 \mathrm{~nm}$ ) as a function of its position (center of nanosphere with respect to substrate surface at $z=0 \mathrm{~nm}$ ). The calculations are performed using the Maxwell stress tensor (MST) method. The nanosphere is centered over the gap between (Au or Si) cylinders. Four different structures are considered: our $\mathrm{Si}$ nanoantenna; two gold cylinders surrounded by a ring (same dimensions as Si nanoantenna) on a glass substrate; two Si cylinders (diameters $200 \mathrm{~nm}$ ) on an Si substrate; and two gold cylinders (diameters $200 \mathrm{~nm}$ ) on a glass substrate. It can be seen that the vertical trapping force pulls the nanosphere toward the antenna. Stable trapping points occur above and at the substrate surface for the Si and Au nanoantennas, respectively. The Au nanoantenna generates a stronger potential than the Si nanoantenna (Fig. 1h), but with severely larger heating (Fig. 1e-f).

\section{Experimental results: optical trapping and tracking of nanoparticles}

We employ fluorescence microscopy to visualize the optical trapping of polystyrene nanospheres (NSs) by our Si nanoantennas. In Fig. 2a, a typical image of our fluorescence microscope is presented, showing two NSs (each 20 $\mathrm{nm}$ diameter) near our Si nanoantenna. In Fig. 2b-d, we plot intensity counts integrated over a square region centered over the Si nanoantenna of each EM-CCD frame as a function of time. The trapping laser is switched off and on at times that are indicated by (orange) upward- and (red) downward-pointing arrows, respectively. Step-like increases in intensity indicate the trapping of individual NSs. In Fig. 2e, we present scatter plots of NS (20 nm) position, collected with a frame rate of 30 fps over a period of 33 seconds for each illumination intensity. The NS positions are found with a radial-symmetry-based particle localization algorithm [6], and illustrate the suppression of Brownian motion with increased trapping laser intensity. The corresponding full-widths-at-half-maximum (FWHMs) of fitted Gaussian distributions are plotted as Fig. 2f. While the potential produced by our Si nanoantenna is not parabolic, for comparison purposes we plot in Fig. $2 \mathrm{~g}$ the "effective" trapping stiffness $\left(k_{\text {eff }}=k_{B} T / \operatorname{var}(x)\right)$. This is extracted from variance of the position (i.e. FWHMs in Fig. 2f) using the method of Ref [7] that accounts for video-image motion blur. In Fig. 2h, we plot the measured fluorescence as a function of trapping laser intensity.
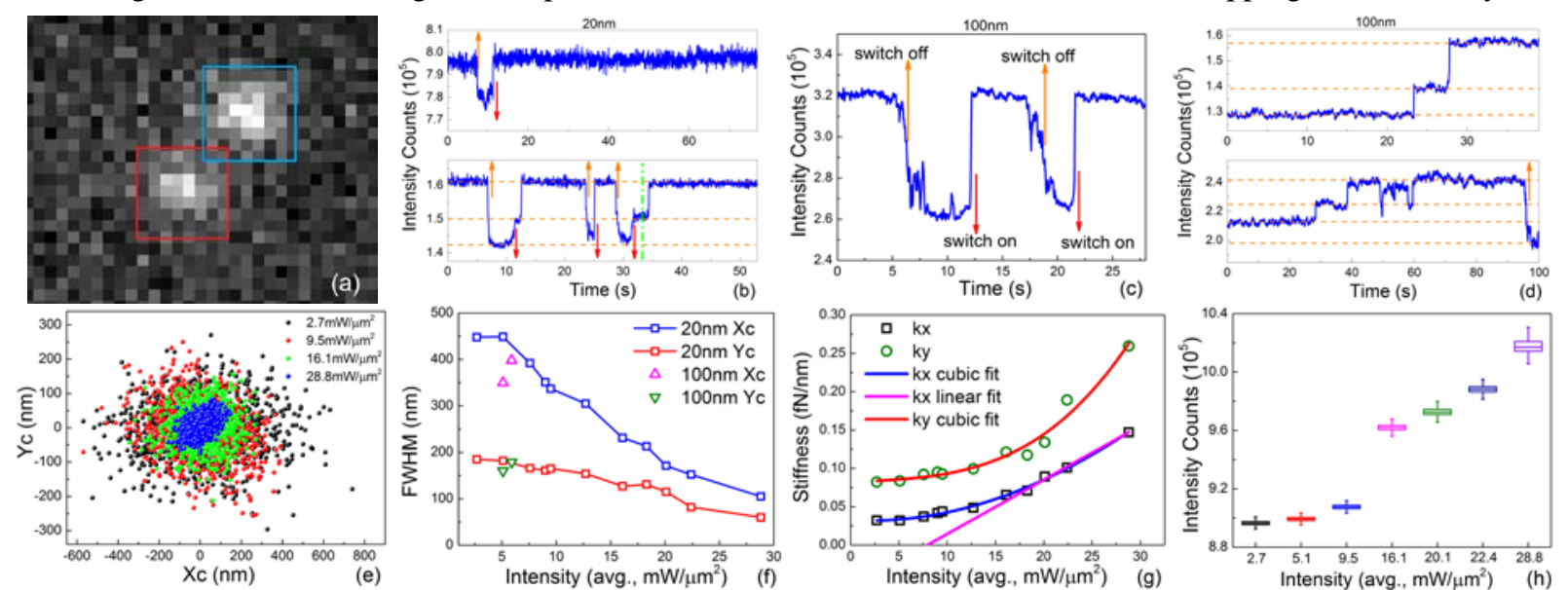

Fig.2. (a) EM-CCD frame showing two nanospheres (NSs, $20 \mathrm{~nm}$ ) near silicon nanoantenna $\left(\mathrm{I}=9 \mathrm{~mW} / \mu \mathrm{m}^{2}\right)$. Red \& blue boxes show trapped and non-trapped nanospheres. Fluorescence intensity vs time for (b) trapping one \& two NSs (20 nm), (c) trapping one NS (100 nm) and (d) trapping one, two \& three NSs $(100 \mathrm{~nm})$. (e) Scatter plots of NS $(20 \mathrm{~nm})$ position. (f) FWHMs of Gaussian fits to position scatter plots vs laser intensity. (g) Motion blur calibrated trapping stiffness vs laser intensity (20 nm NS). (h) Fluorescence counts vs trapping laser intensity (20 nm NS).

\section{References}

[1] M.L. Juan, M. Righini, and R. Quidant. Nature Photonics 5, 349 (2011)

[2] J.S. Donner, G. Baffou, D. McCloskey, and R. Quidant. ACS Nano 5, 5457 (2011)

[3] K. Wang, E. Schonbrun, P. Steinvurzel, and K. B. Crozier. Nature Communications 2, 469 (2011)

[4] A. E. Krasnok, A. E. Miroshnichenko, P. A. Belov, and Y. S. Kivshar. Optics Express 20, 20599 (2012)

[5] M. Caldarola et al. Nature Communications 6, 7915 (2015)

[6] R. Parthasarathy. Nature Methods 9, 724 (2012)

[7] W. P. Wong, and K. Halvorsen. Optics express 14, 12517 (2006) 